\title{
Structural basis of prokaryotic NAD-RNA decapping by NudC
}

Cell Research (2016) 26:1062-1066. doi:10.1038/cr.2016.98; published online 26 August 2016

\section{Dear Editor,}

In eukaryotes, the 5',5'-triphosphate-linked 7-methylguanosine $\left(\mathrm{m}^{7} \mathrm{G}\right)$ cap is essential for modulation of mRNA metabolism and protects the mRNA from degradation [1]. Removal of the cap from the $5^{\prime}$-terminus of the RNA is mainly carried out by decapping protein 2 (Dcp2) $[2,3]$ and scavenger decapping enzyme (DcpS) [4]. A distinctive feature of prokaryotic mRNA is the absence of a 5'-capped structure. Recently, nicotinamide adenine dinucleotide (NAD) or a triphosphate group has been shown to be covalently linked to the 5 '-end of certain types of prokaryotic RNAs [5, 6] as a cap that improves stability, and NADH pyrophosphatase (NudC) [6] and RNA pyrophosphohydrolase $(\mathrm{RppH})$ [7] are responsible for the $5^{\prime}$ hydrolysis of NAD or the triphosphate group, respectively, from the RNA. Interestingly, Dcp2, NudC and RppH all belong to the Nudix hydrolase superfamily, whose members act on substrates of nucleoside diphosphate linked to another moiety, X (hence the name Nudix) [8]. The Nudix enzymes share a conserved 23-amino acid sequence termed the Nudix motif $\left(\mathrm{GX}_{5} \mathrm{EX}_{7} \mathrm{REUXEEXGU}\right)$, which is required for substrate catalysis, but each uses a distinct strategy for substrate recognition. Although the molecular bases of substrate recognition by Dcp2 and RppH have been well studied [9, 10], the mechanism by which NudC removes the NAD cap still remains to be elucidated. Here, we report the crystal structure of Escherichia coli NudC in complex with NAD, uncovering the structural basis of NudC-mediated decapping of NAD-capped RNA.

We purified recombinant NudC proteins to homogeneity and tested their NAD hydrolysis activity by using high-performance liquid chromatography (HPLC). NudC hydrolyzed NAD in the presence of $\mathrm{Mg}^{2+}$, generating nicotinamide mononucleotide (NMN) and adenosine 5'-monophosphate (AMP); however, a NudC active-site mutant (E178Q) showed almost undetectable hydrolytic activity towards NAD (Figure 1A). Thus, we used this mutant to crystallize the substrate-bound NudC complex. After numerous unsuccessful trials, we finally crystallized NAD- bound NudC (residues 1-257, E178Q) in space group $P 22_{1} 2_{1}$. On the basis of the two available coordinates of NAD-free NudC (PDB: 2GB5 and 1VK6), the structure of the NAD-NudC complex was determined by molecular replacement and refined to $2.4 \AA$ resolution (Supplementary information, Table S1). After the assignment of most of the NudC amino acids into the electron density map, electron densities indicative of the NAD molecule were clearly visible (Supplementary information, Figure S1). In each asymmetric unit, two protein molecules bound two NAD molecules and formed a symmetric homodimer. The two NudC subunits adopt an almost identical conformation, with a root-mean-square deviation (RMSD) of $0.41 \AA$ over $234 \mathrm{C} \alpha$ atoms (Supplementary information, Figure S2A). A previously deposited NADfree structure (PDB: 2GB5) also exists as dimer, and superposition of dimers in the NAD-free state and in the NAD-bound state generated an RMSD value of $0.46 \AA$ over $478 \mathrm{C} \alpha$ atoms (Supplementary information, Figure S2B), thus suggesting that NudC undergoes little conformational change upon NAD binding.

The NudC structure shows a conformation of a tight symmetric homodimer with the appearance of a butterfly with a wingspan of $\sim 70 \AA$ and a height of $60 \AA$ (Figure 1B). The dimer interface, which has an area of $\sim 3300$ $\AA^{2}$, is primarily formed by polar contacts, water-mediated hydrogen bonds, and Van der Waals interactions. The interface contains 22 residues from each protomer and consists of two regions, from residues R93 to I127 and E162 to S191 (Supplementary information, Figure S3A); these residues form an extensive interaction network that is difficult to disrupt. To investigate the state of NudC in solution, we performed size-exclusion chromatography (SEC), analytical ultracentrifugation (AUC) and static light scattering (SLS) analyses. The elution profiles of NudC and the E178Q mutant in SEC suggest that the protein likely exists as a dimer (Supplementary information, Figure S3B). The AUC and SLS analyses of NudC indicated molecular masses of $\sim 66.3$ and $63.0 \mathrm{kDa}$, respectively (Supplementary information, Figure S3C and S3D). These results are similar to the exact molecular 
A

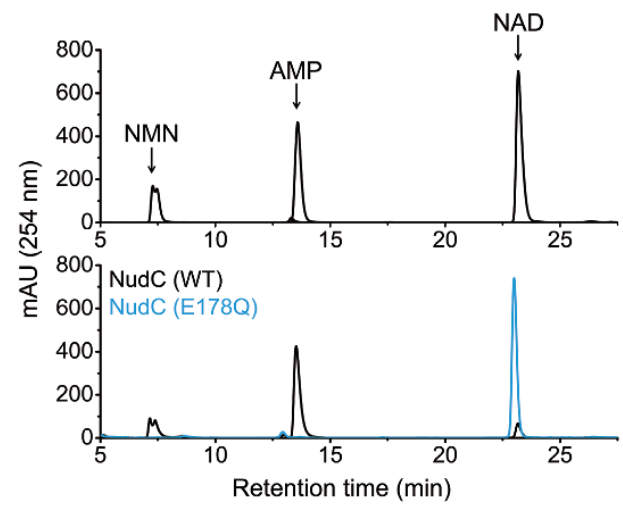

C
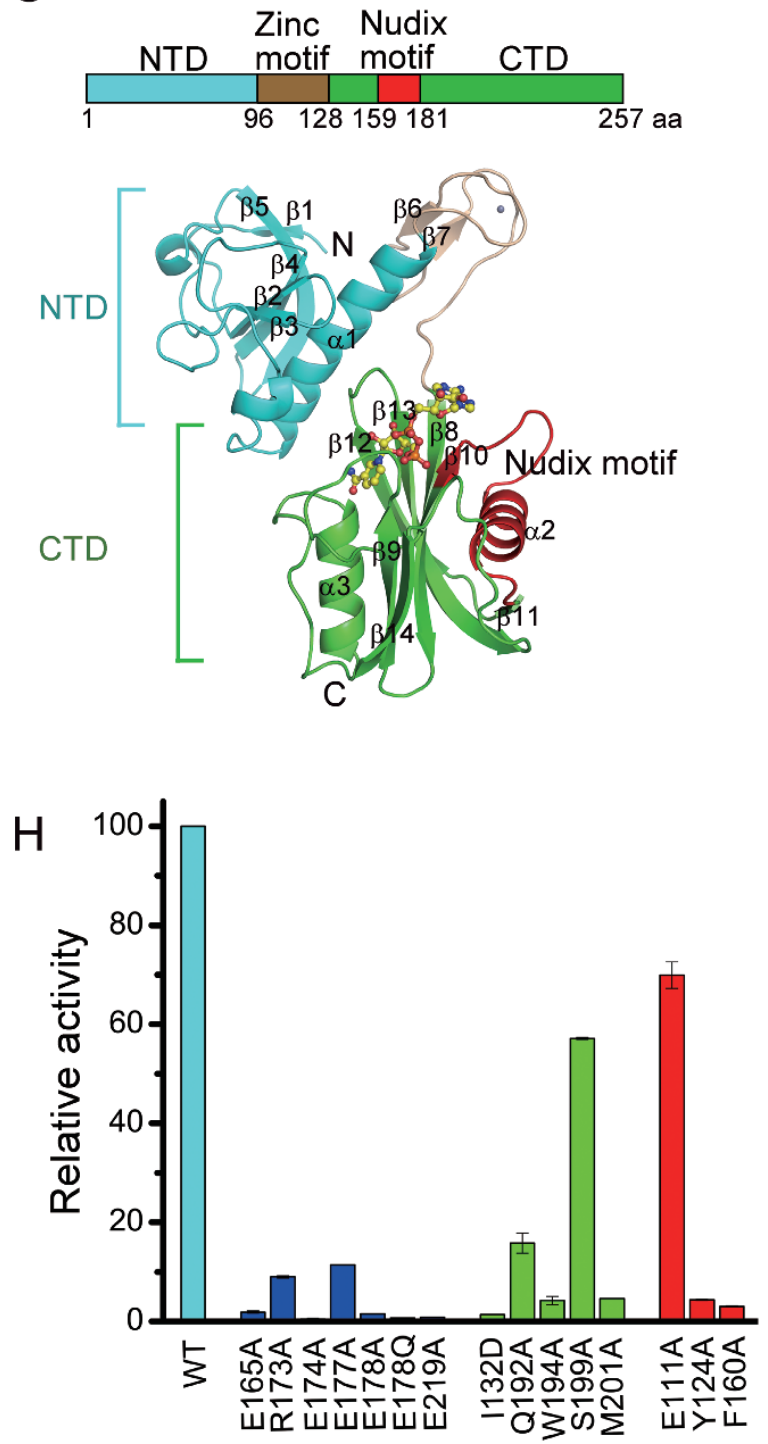

B
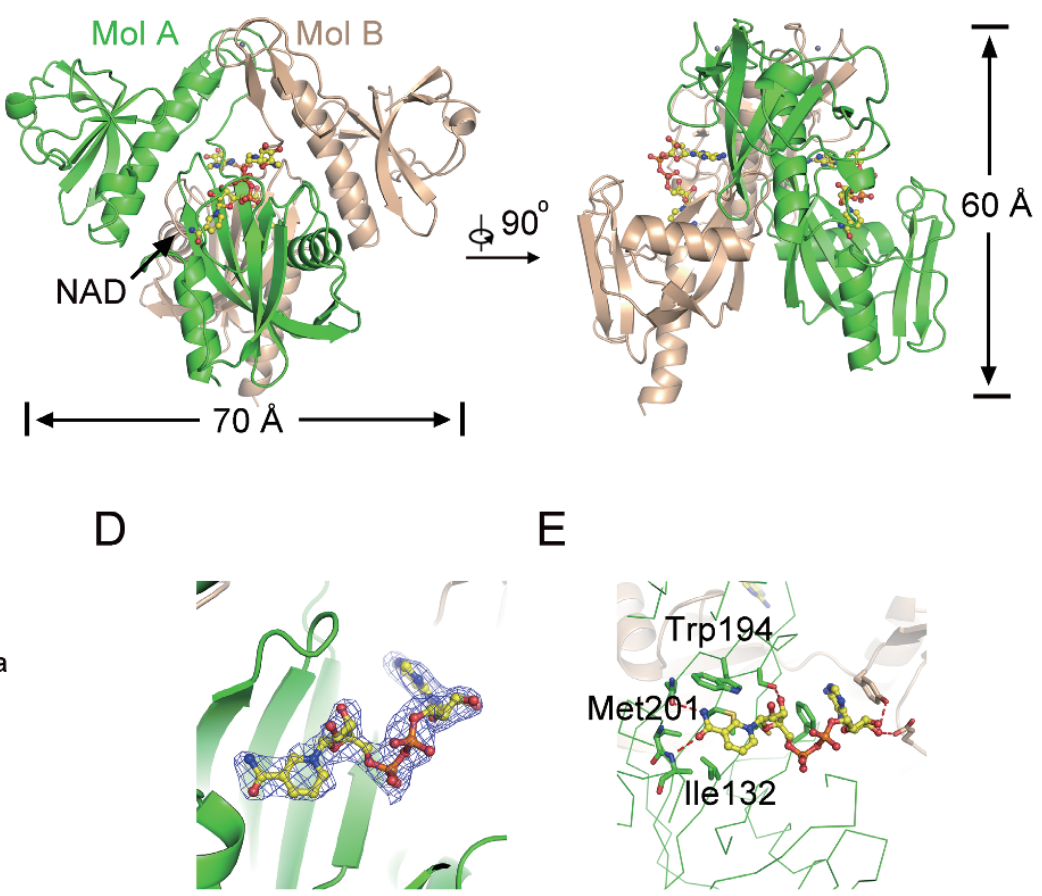

$E$

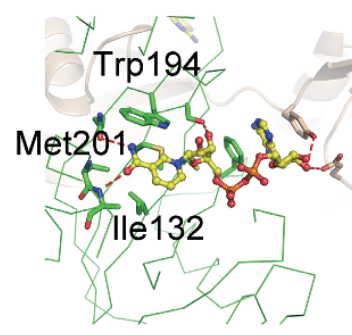

F

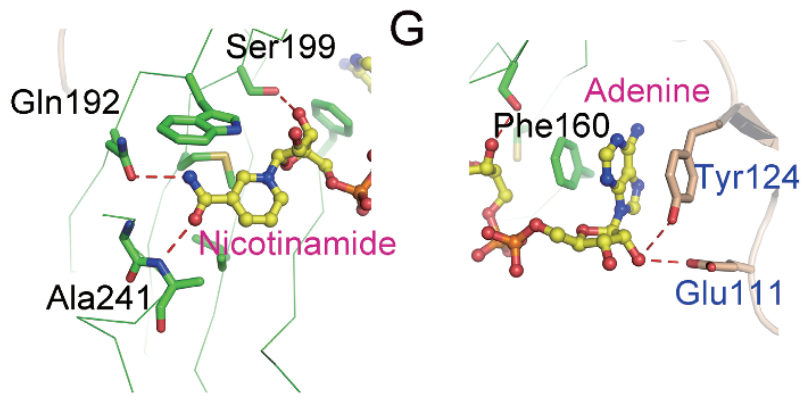

I
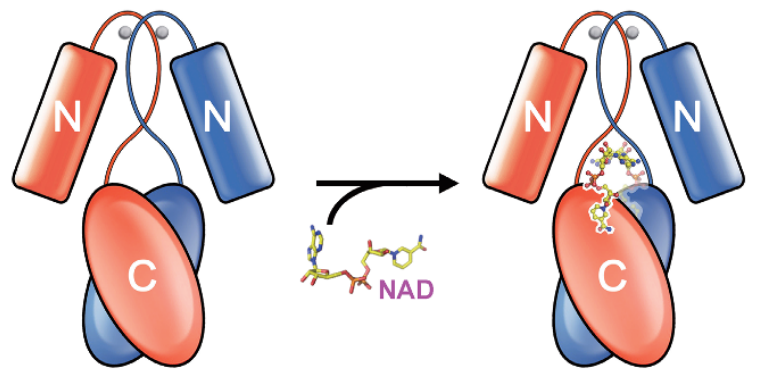
mass of a NudC dimer (65.2 kDa) and are consistent our observations from the crystal structure.

We traced the structure of NudC (Mol A, residues 1-257), consisting of an N-terminal domain (NTD, residues 1-96) and a C-terminal domain (CTD, residues 128257) separated by a zinc motif (residues 97-127; Figure $1 \mathrm{C}$ and Supplementary information, Figure S4). The NTD is swapped between the two NudC subunits of the homodimer, like that observed in the open conformation of apo Nudt7 of Arabidopsis thaliana [11], and includes five $\beta$-strands ( $\beta 1-\beta 5)$, two $3{ }_{10}$-helices $(\eta 1$ and $\eta 2)$ and one $\alpha$-helix $(\alpha 1)$. A Dali search using the NTD as a query showed that 18 of 20 retrieved structures with Z-scores higher than 5.0 were Nudix enzymes (Supplementary information, Table S2). The zinc motif, which participates in NudC dimer formation, contains two anti-parallel $\beta$-strands ( $\beta 6-\beta 7)$ and one zinc ion coordinated with four cysteine residues (Cys98, Cys101, Cys116, and Cys119), of which the first three are highly conserved in NudC homologs (Supplementary information, Figure S5). Consistent with this observation, mutation of any of the conserved cysteines (Cys98, Cys101, or Cys116) resulted in severe protein aggregation, suggesting that the zinc motif has an important role in maintaining the NudC structure.

The CTD adopts a canonical Nudix fold with a seven-stranded mixed sheet ( $\beta 8-\beta 14)$ sandwiched between two remote perpendicular $\alpha$-helices ( $\alpha 2$ and $\alpha 3$ ). The conserved Nudix motif (residues 159-181) contains the catalytic residues E174, E177 and E178, which are located in a loop-helix-loop structure formed by helix $\alpha 2$ and the adjacent loops (Figure 1C and Supplementary information, Figure S5). Alignment of the NudC structure with the decapping enzymes RppH and Dcp2 revealed that the NudC CTD is similar to the corresponding domains of RppH and Dcp2 (Supplementary information, Figure S6A), and the Nudix motifs of the enzymes could nearly be superposed. These results suggest that the catalytic mechanism of NudC is similar to those of Dcp2 and $\mathrm{RppH}$, which share a water-mediated and metal-dependent pyrophosphate hydrolysis model $[10,11]$. Despite the similarity in the overall conformation of the CTD, each of the C-terminal $\alpha$-helices in these enzymes adopts different orientations, suggesting an essential role in substrate recognition (Supplementary information, Figure S6B).

The NAD binding pocket in NudC is pre-organized, which was observed by comparing the surface view of NudC in the NAD-free and NAD-bound states (Supplementary information, S7A). The NAD molecule is mainly accommodated by the NudC CTD (Figure 1D), and the NAD-NudC recognition is accomplished in two major steps. First, the nicotinamide moiety is trapped in a cavity mainly comprising several bulky hydrophobic residues, including I132, W194 and M201 (Figure 1E). The amide nicotinamide group forms two hydrogen bonds with the carbonyl oxygen of Q192 in $\beta 12$ and the main chain of A241 in the C-terminal helix, and a subtle change in the orientation of this helix would break the latter hydrogen bond. The 3-hydroxyl group of the nicotinamide ribose interacts with S199 (Figure 1F). In a previous report, residues Q192, W194, and S199 have been found to lie within a conserved eight-amino acid motif (SQPWPFPXS), which has been used to classify NudC and other Nudix enzymes into the NADH hydrolase subfamily [12], and the observed interactions here are consistent with the conservation of these residues.

Figure 1 Structure of the NAD-NudC complex and recognition of NAD by NudC. (A) NudC-mediated hydrolysis of NAD was detected by high-performance liquid chromatography (HPLC). The elution profiles of the standard samples NMN, AMP and NAD were recorded at $254 \mathrm{~nm}$ and are shown in the upper panel. The lower panel shows the elution profiles of NAD processed by wild-type NudC and the E178Q mutant. (B) Overall structure of the NAD-NudC complex in two perpendicular views. The NudC homodimer consists of two symmetric subunits ( $M o l ~ A$ in green and Mol $B$ in wheat) and resembles a butterfly with a wingspan of approximately $70 \AA$ and a height of $60 \AA$. Each subunit binds one NAD molecule. NAD is shown in a yellow ball-and-stick model and is indicated by an arrow. Zinc ions are represented by gray spheres. (C) A ribbon representation of the Mol A subunit. The N-terminal domain (NTD) is colored cyan, the zinc-motif is colored bronze, the C-terminal domain (CTD) is colored green, and the Nudix motif within the CTD is colored red. The numbers under the column depict the boundaries of the motifs and domains in NudC. The labels on the helices or strands were used to trace the NudC sequence. (D) The 2Fo-Fc electron density of NAD, contoured at $1 \sigma$ and shown in blue, is clearly visible in the cavity of the CTD. (E) Closeup view of the NAD binding site. The residues participating in the interactions with NAD are shown as sticks, with nitrogen in blue and oxygen in red. The hydrogen bonds are represented by red dashed lines. The key residues of the hydrophobic cavity that accommodates the NAD nicotinamide moiety are labeled. (F) The recognition of the NAD nicotinamide moiety by NudC. The residues that mediate the interactions with NAD via hydrogen bonds are labeled. (G) The adenine moiety of NAD is stacked with the phenyl rings of F160 on Mol A and Y124 on Mol B. Two hydrogen bonds buttress the stacking effect. (H) The NAD hydrolysis activity of NudC and the mutants. The activity was measured by HPLC. Each experiment was independently repeated three times; average values and standard deviations are shown. (I) Model representation of formation of the NAD-NudC complex. 
Second, the adenine base is stacked via $\pi-\pi$ interactions with the benzene rings of F160 from Mol A and Y124 from Mol B (Figure 1G). Buttressing the stacking interactions with the adenine base, the 2-hydroxyl group of the adenine ribose interacts with E111 and Y124 from Mol B. These observations suggest the essential role of NudC dimerization in substrate recognition, which is reminiscent of the substrate binding mode of ADP-ribose pyrophosphatase [13].

We mutated the relevant residues and examined their effects on NudC-mediated NAD hydrolysis (Figure $1 \mathrm{H})$ to corroborate the structural findings. As expected, a series of charged residue substitutions in the Nudix motif significantly diminished enzyme activity. The single-residue mutants I132D, W194A and M201A retained little catalytic activity, indicating that the integrity of the hydrophobic substrate-binding cavity is highly important for NAD recognition by NudC. In addition, breakage of the hydrogen bond mediated by Q192 markedly reduced the catalytic activity of NudC, and the stacking effect between residues F160/Y124 and the adenine base of NAD was also essential for substrate coordination, because F160A and Y124A mutants were nearly inactive. Neither the E111A nor the S199A mutation had a substantial effect on NudC activity, suggesting a minor role for the NAD ribose moiety in substrate-NudC recognition.

Similar results were observed for the cleavage of 5'-NAD-capped RNA by NudC; the substrate was cleaved into NMN and monophosphorylated RNA (Supplementary information, Figure S7B). As anticipated, most of the alanine substitutions in residues responsible for NAD recognition, such as Y124, I132, F160, W194, and M201, and key residues that participate in catalysis, such as E165, R173, E174, E178, and E219, significantly diminished the cleavage of NAD-capped RNA (Supplementary information, Figure 7C). In addition, the E177A and Q192A mutants exhibited half of the wild-type activity. Altogether, these results suggest that RNA recognition by NudC occurs mainly through binding with the NAD moiety. Intriguingly, most of the residues involved in NAD recognition are highly conserved in NudC homologs ranging from E. coli to human (Supplementary information, Figure S5). Given the prevalence of NAD in the three kingdoms, the NAD capping/decapping processes for RNA might be evolutionarily conserved. To examine this possibility we investigated the ability of several NudC homologs to cleave NAD-capped RNA. NPY1 and Nudt19, the NudC homologs from Saccharomyces cerevisiae and Oryza sativa, respectively, hydrolyzed the substrate into NMN and monophosphorylated RNA in vitro (Supplementary information, Figure S7D). Although further investigation is required, these results raise the possibility that NAD-mediated RNA processing might be a universal mechanism that also operates in higher eukaryotic species. A recent finding that both bacterial RNAP and eukaryotic RNAP II show the ability of incorporating the NAD cap into RNA [14] corroborates our speculation.

The RNA decapping process is precisely regulated in vivo, as exemplified by the Dcplp-regulated hydrolytic activity of Dcp2p on $\mathrm{m}^{7} \mathrm{G}$-capped RNA [2] and the DapF-regulated pyrophosphatase activity of $\mathrm{RppH}$ [15]. A structural investigation showed that Dcplp binds to the NTD of Dcp2p and stimulates its activity [2]. These findings raise the possibility that the decapping activity of NudC may also be regulated by other factors through interaction with the NTD.

In summary, our data not only reveal the molecular basis of NAD-capped RNA recognition by the NudC dimer (Figure 1I) but also provide the framework for further functional identification of regulatory factors involved in the RNA decapping process. The architecture of the NAD-NudC complex also provides a structural explanation for the conservation of residues that might participate in recognition of NAD and NAD-capped RNA in organisms ranging from $E$. coli to human. Moreover, the ability of the two examined NudC eukaryotic homologs to cleave NAD-capped RNAs suggests that the process of removing the NAD-cap from the 5'-terminus of RNA is evolutionarily conserved.

The atomic coordinates and structure factors for the structure have been deposited in the Protein Data Bank (PDB) with the accession code 5ISY.

\section{Acknowledgments}

We thank J He at Shanghai Synchrotron Radiation Facility (SSRF) beamline BL17U, and R Zhang at SSRF beamline BL19U for on-site assistance. We thank S Fan at the College of Life Science, Tsinghua University, for data collection support. We thank research associates at Center for Protein Research (CPR) and State Key Laboratory of Agricultural Microbiology, Huazhong Agricultural University, for technical support. This work was supported by funds from the Ministry of Science and Technology (grant number 2015CB910900), Fok Ying-Tong Education Foundation (grant 151021), the Fundamental Research Funds for the Central Universities (Program No. 2014PY026, No. 2015PY219, and No. 2014JQ001), and Huazhong Agricultural University Scientific \& Technological Self-innovation Foundation (Program No. 2013RC013).

Delin Zhang ${ }^{1, *}$, Yexing Liu ${ }^{2, *}$, Qiang Wang ${ }^{1}$, Zeyuan Guan ${ }^{1}$, Jing Wang ${ }^{1}$, Jian Liu ${ }^{1}$, Tingting Zou ${ }^{1,3}$, Ping Yin ${ }^{1}$

${ }^{I}$ National Key Laboratory of Crop Genetic Improvement and National 
Centre of Plant Gene Research, Huazhong Agricultural University, Wuhan 430070, China; ${ }^{2}$ Center for Structural Biology, School of Life Science, Tsinghua University, Beijing 100084, China; ${ }^{3}$ College of Life Sciences and Technology, Huazhong Agricultural University, Wuhan 430070, China

*These two authors contributed equally to this work.

Correspondence: Ping Yin

E-mail: yinping@mail.hzau.edu.cn

\section{References}

1 Coller J, Parker R. Аnпи Rev Biochem 2004; 73:861-890.

2 She MP, Decker CJ, Svergun DI, et al. Mol Cell 2008; 29:337-349.

3 Wang ZR, Jiao XF, Carr-Schmid A, et al. Proc Natl Acad Sci USA 2002; 99:12663-12668.

4 Wang ZR, Kiledjian M. Cell 2001; 107:751-762.

5 Chen YG, Kowtoniuk WE, Agarwal I, et al. Nat Chem Biol 2009; 5:879-881.

6 Cahová H, Winz M, Höfer K, et al. Nature 2014; 519:374-377.

7 Deana A, Celesnik H, Belasco JG. Nature 2008; 451:355-358.

8 McLennan AG. Cell Mol Life Sci 2006; 63:123-143.

9 Valkov E, Muthukumar S, Chang CT, et al. Nat Struct Mol Biol 2016; 23:574-579.

10 Piton J, Larue V, Thillier Y, et al. Proc Natl Acad Sci USA 2013;
110:8858-8863.

11 Tang QY, Liu CF, Zhong C, et al. Mol Plant 2015; 8:1557-1559.

12 Dunn CA, O'Handley SF, Frick DN, et al. J Biol Chem 1999; 274:32318-32324.

13 Gabelli SB, Bianchet MA, Bessman MJ, et al. Nat Struct Biol 2001; 8:467-472.

14 Bird JG, Zhang Y, Tian Y, et al. Nature 2016; 535:444-447.

15 Lee CR, Kim M, Park YH, et al. Nucleic Acids Res 2014; 42:1274612757.

(Supplementary information is linked to the online version of the paper on the Cell Research website.)

cc)(-)(2) This license allows readers to copy, distribute and transmit the Contribution as long as it attributed back to the author. Readers are permitted to alter, transform or build upon the Contribution as long as the resulting work is then distributed under this is a similar license. Readers are not permitted to use the Contribution for commercial purposes. Please read the full license for further details at - http://creativecommons.org/ licenses/by-nc-sa/4.0/

(C) The Author(s) 2016 